EPJ Web of Conferences 47, 10003 (2013)

DOI: $10.1051 /$ epjconf/20134710003

(C) Owned by the authors, published by EDP Sciences, 2013

\title{
Derivation of the parameters of CoRoT planets
}

\author{
J. Cabrera ${ }^{1, a}$, Sz. Csizmadia ${ }^{1}$, Th. Pasternacki ${ }^{1}$, C. Dreyer ${ }^{1}$, A. Erikson ${ }^{1}$ \\ and $\mathrm{H}$. Rauer ${ }^{1,2}$ \\ ${ }^{1}$ Institute of Planetary Research, German Aerospace Center, Rutherfordstrasse 2, \\ 12489 Berlin, Germany \\ 2 Zentrum für Astronomie und Astrophysik, TU Berlin, Hardenbergstrasse 36, 10623 Berlin, \\ Germany
}

\begin{abstract}
We explore the influence that limb darkening and stellar activity have in the determination of planetary parameters, highlighting the impact that they have in space-based surveys, such as CoRoT.
\end{abstract}

\section{INTRODUCTION}

The simultaneous measurement of the mass and the radius of an extrasolar planet provides the first information about its internal structure and composition, which is later needed to understand its formation and evolution. In this work we study the derivation of the radius of a transiting extrasolar planet from the analysis of its photometric light curve. In particular, we discuss the impact that stellar limb darkening has when obtaining the value of the relative radius between the star and the planet and the systematic uncertainties created by stellar activity. The interested reader can obtain detailed information about transiting extrasolar planets from $[1,2]$ and references therein.

Transit parameters are determined by fitting a theoretical model to the observed light curve observations. A precise fit is a nonlinear, multi-dimensional problem which has been solved by different authors in many ways. For example, one can use elliptic functions to describe the light curve shape [3]; or Jacobi polynomials [4, 5]; or semi-analytical approximations, such as EBOP [6, 7] or JKTEBOP [8]. There are also fully numerical codes, mostly used for eclipsing binary stars [9-15]. Finally, the fit model has to be coupled with an optimization code. Most popular methods are MCMC, Amoeba, and genetic algorithms. For example, sometimes Amoeba is used for optimization, and later MCMC for deriving the uncertainties [16]. TLCM [17] uses genetic algorithms for minimization and Simulated Annealing for deriving uncertainties. The later code is routinely used for CoRoT exoplanets, but there are more approaches, a recent case being EXOFAST, which uses a differential evolution MCMC code [18].

\section{DERIVATION OF THE PLANETARY PARAMETERS}

Once the signal of a transiting planet has been detected, there is an optimal strategy to assess the reliability of the signal to the follow-up procedure that will lead to the final confirmation of the candidate as a planet. These procedures have been described by different authors; see, for example, [19-22]. What the light curve modeling provides at these stage are a first estimation of the planetary parameters relative to the stellar ones, namely the radius ratio $k=R_{\mathrm{p}} / R_{\mathrm{s}}$, the relative semi-major axis $a_{\mathrm{p}} / R_{\mathrm{s}}$,

\footnotetext{
ae-mail: juan.cabrera@dlr.de
}

This is an Open Access article distributed under the terms of the Creative Commons Attribution License 2.0, which permits unrestricted use, distribution, and reproduction in any medium, provided the original work is properly cited. 
the impact parameter $b$, the limb-darkening coefficients, the photometric contamination by third light, and information about the epoch of the transit (useful for the study of transit timing variations). Next steps involve spectroscopic characterization of the host star to reject false alarms (see [23, 24]) and to calculate absolute values for the relative planetary parameters obtained from the light curve analysis. Stellar parameters obtained from spectroscopic analysis are model dependent, however transiting planets provide an useful constrain for those models: the stellar $\log g$ from the transit fit (see the discussion in [25] and, in this volume, the contribution from J. Valenti). However, current models include a certain uncertainty in the stellar parameters, which are typically larger than $10 \%$ for the mass and the radius [25]. Asteroseismology can overcome these limitations, but it is only feasible from space for bright targets (see [26-28] and, in this volume, the contribution from A. Moya). The uncertainty in the stellar parameters is directly translated into the uncertainty of the planetary parameters, where precisions of a few percent are required to distinguish between different compositions and internal structures [29-35].

\section{THE IMPACT OF LIMB DARKENING}

Figure 1 shows the values that different authors get for the quadratic limb darkening coefficients for stars of different effective temperatures. It is known from previous studies (see [36, 37] and references therein) that theoretical values deviate significantly from each other among different models depending on which temperature region we consider. Some transit modelers fix the limb darkening coefficients according to tables when they fit a transit curve. As is clear from the aforementioned discrepancies, in some temperature regions this is not a good strategy because the values of the limb darkening coefficients are not consistent and it is not clear which are the correct ones. Moreover, in the modeling of transits, limb darkening coefficients are strongly correlated to other parameters, for example the impact parameter $b$ or the radius ratio $k$ (see, for illustration, Figure 4 from [17]). Therefore, if due to the uncertainties in the stellar parameters or in the theoretical models, the limb darkening coefficients are fixed to an incorrect value, the derived planetary parameters will suffer of a systematic uncertainty difficult to evaluate. Although sometimes theory and observations agree well (see the examples of CoRoT-8b [38] or CoRoT$11 \mathrm{~b}$ [39]), in numerous cases authors have noticed significant discrepancies (see the cases of CoRoT12b [40], CoRoT-13b [41, 42], HD 209458b [43], Kepler-5b [16], or WASP-13b [44]). Although recent improvements have been made towards a more detailed modeling of stellar atmospheres (see [45]), the current status of our knowledge of stellar parameters advocates for a conservative use of any priors on the limb darkening coefficients based on the spectroscopic analysis of the star. [37] made a quantitative analysis of how the uncertainties in the limb darkening coefficients translate into the uncertainties of the planetary parameters, such as the radius ratio $k$. They conclude that for stars similar to the Sun, where most models agree with each other, typical uncertainties are comparable to those coming from other sources. However, for cool stars, the uncertainties in the limb darkening coefficients become dominant and produce unacceptable uncertainties in the planetary parameters, specially when we consider the photometric accuracy of space-based surveys such as CoRoT and Kepler.

\section{THE IMPACT OF STELLAR ACTIVITY}

The result of a fit to the transit light curve with adjustale limb darkening coefficients can lead to unreasonable limb darkening values. For example, [42] accepted a worse solution for CoRoT-13b in the sense of the quality of the fit (measured by the $\chi^{2}$-values), because when they left free both limb darkening coefficients, they obtained unphysical values, i.e. far from the theoretically predicted range, sometimes causing limb brightening instead of limb darkening or negative fluxes at the edge. What can we say about such cases?

We would like to point out that we employed an indirect assumption in the previous sections: the effective temperature of the stellar surface is assumed to be the same at each point of the surface and therefore the stellar surface brightness distribution is only modified by limb darkening. The same 


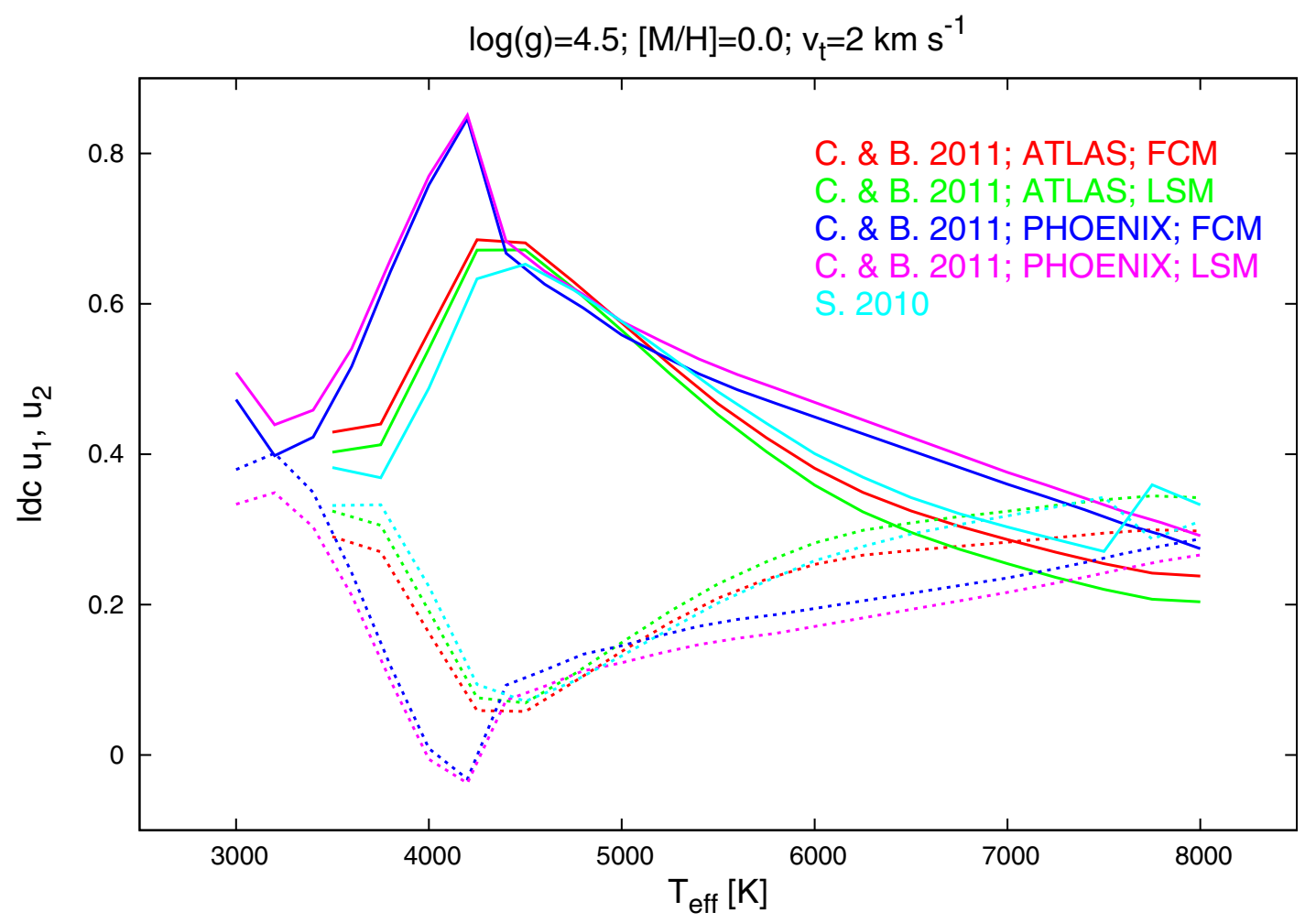

Figure 1. Comparison of the values of the quadratic limb darkening coefficients for different theoretical models as a function of the stellar effective temperature. For the sake of correct comparison, we used the same $\log g$ and turbulent velocity $v_{t}$ values for all the curves. Solid lines represent the $u_{1}$ coefficients, dotted lines the $u_{2}$ coefficients. The model of [46] is denoted by cyan lines. Red lines show the models of [47], which are based on the ATLAS synthetic spectra and their flux conservation method (FCM). Green lines were obtained by them using the same spectra, but applying their least square method (LSM). Violet and magenta lines use the same methods as the two previous ones, but for PHOENIX models. The figure only hows the CoRoT white light pass band coefficients; the discrepancies are similar for Kepler's response function and for other photometric systems. Figure adapted from [37].

assumption is used when the theoretical limb darkening coefficients are calculated, and almost every transit light curve investigator uses the same assumption. However, there are several factors that modify the local effective temperature on the stellar surface. Such effects are e.g. stellar rotation (i.e. gravity darkening, see e.g. [48-52], reflection effect (although we can neglect this for the star in a star-planet system), and stellar activity phenomena: spots, plage-area, faculae and flares. Some of these are transient phenomena, e.g. the flares, but others have similar or much longer timescales than the length of the transits (e.g. the spots and faculae, [53]). They can be regarded as an additional, but constant surface brightness distortion during a transit. Constant here means that we assume that the number, temperature, size, astrographic longitude, and latitude of the distortions do not change around and during a transit. The constancy of the aforementioned quantities is reasonable.

These distortions mean that the local effective surface temperature where they occur is lower for stellar spots and higher for faculae than that of the undistorted stellar surface. As it is well known, the limb darkening coefficient is different for different surface temperatures. This means that the apparent stellar disc cannot be characterized by only one effective surface temperature value, and that is why the surface brightness distribution cannot be characterized by only using the limb darkening coefficients due to only one effective temperature. 

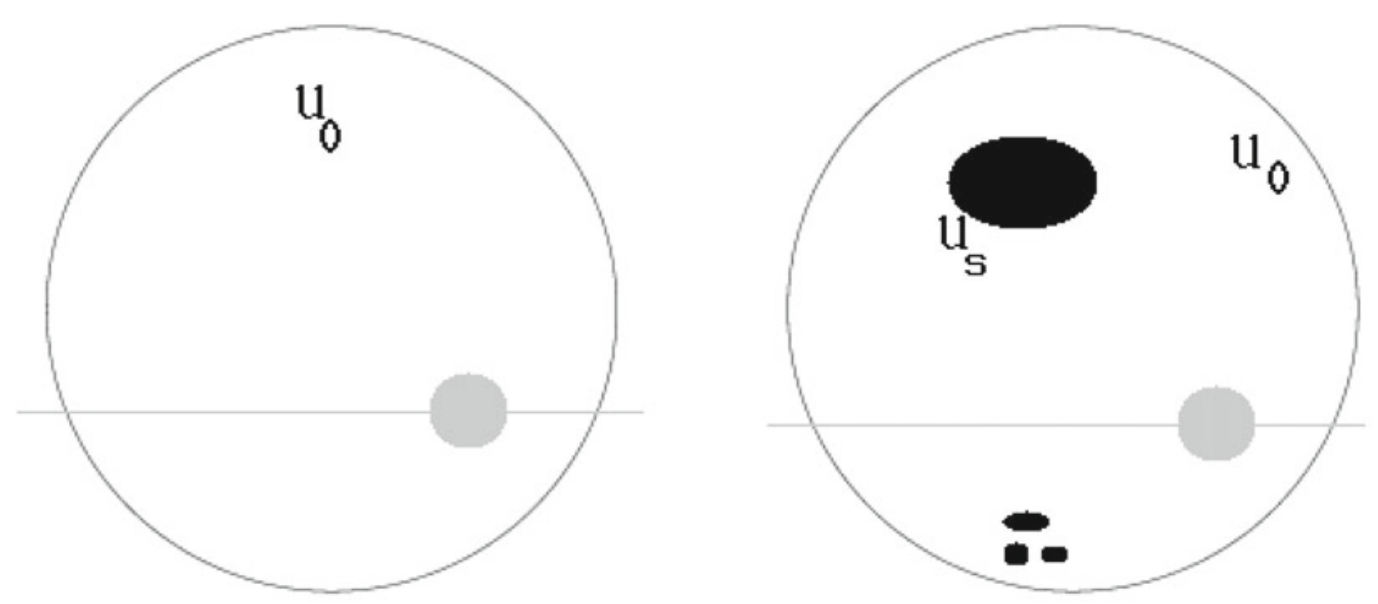

Figure 2. Illustration of the effect of Type I spots. Left: the planet crosses an unmaculated star that is characterized with some limb darkening coefficient $u_{0}$. Right: the planet crosses the apparent stellar disc of a spotted star, where the spots and the planet have different impact parameters, as well as the stellar photosphere and the spots have different limb darkening coefficients $\left(u_{0}, u_{s}\right)$. Grey area is the planet, black ellipses represent the spots. Figure adapted from [37].

[12] already took into account that the undistorted and the distorted areas have different limb darkening coefficients, when he developed a code to analyze eclipsing binary star light curves. The corresponding limb darkening coefficients were taken from theoretical approximations available at that time. We would need a similar approach for the transit modeling. However, we suggest avoiding exactly the same approach for the following reasons:

a) Different tables give different limb darkening coefficients, which is why the results can be different, and maybe the derived spot parameters will depend on the assumed limb darkening coefficients;

b) The spot distribution is not well known, and the spot solution is often highly degenerated so the reliability of the spot solution can be criticized if only one-color photometry is available. Consequently it is hard to include the spots in a modeling tool.

To investigate the effect of stellar spots and faculae (hereafter we will use the term spot for both cases, considering that spots having higher temperatures than the surface, i.e. "bright spots", describe the characteristics of faculae, which have a higher temperature than the normal photospheric area), we classify the spots as follows. Spots that are not eclipsed by the planet we define as Type I spots and spots that are eclipsed by the planet as Type II spots.

The effect of Type II spots can be taken into account because the size and location of the spots can be determined with high accuracy, and that is why removing their contribution from the light curve causes no problem, at least in principle [54-56].

Type I spots are more complicated. Those that cause light curve modulations can be removed safely with baseline fits and baseline corrections using the out-of-transit points in the small vicinity of transits. But there are also spots that cause no light curve modulations, e.g. a polar spot. In addition, [57] propose, based on spectral evidence, that a considerable amount of stars exhibit numerous (up to $\sim 5000$ ), small (not bigger than 2-3 astrographic degrees) dark spots that are axysymmetrically distributed on the stellar surface, reaching $\sim 50 \%$ spot-coverage, which leads to no observable light curve modulation over the current detection limit in spite of their high activity level. If this is true then we can expect that even photometrically quiet stars can produce strange limb darkening coefficients as we will show.

Figure 2 illustrates the situation. One can calculate how the observable flux changes when the planet moves in front of a spotted star and compare it with the case of the same planet orbiting an unmaculated stellar surface (see [37]). Although the transit depth will change due to the presence of stellar spots, 


\section{Hot Planets and Cool Stars}

it is easy to show that the radius ratio $k$ remains unchanged and precisely determinable if one chooses the effective limb darkening coefficients appropriately. Spots act like a contamination source in the aperture. If an unresolved star or other light source (also called "contamination source") contributes to the observed flux, then that decreases the observed transit depth. This extra light source is routinely removed from the light curve in general.

It has been already recognized that Type I spots can act as contamination sources, but our result implies also that limb darkening coefficients can also change. This problem however can be solved in the following simple and robust way: limb darkening coefficients and contamination factor should be free parameters. Last but not least it shows that the observed limb darkening coefficients can be highly different from the theoretically predicted values in the presence of spots, even if they do not produce light curve modulations. The uniqueness of the transit light curve solutions is determined by the fact that the length of the ingress and egress phases, as well as the full transit length, are also related to the size ratio of the star and the planet.

\section{SUMMARY}

Precise planetary parameters are required to improve our knowledge of the interior structure and composition of extrasolar planets. In the long term, we aim at performing statistical studies of planetary properties, which would lead to the first studies of exoplanetology, as we do today in the Solar System. Obtaining these precise parameters requires a careful observational strategy (see Section 2) which considers stars and planets as systems instead of separate objects, and a careful understanding of the observational and theoretical uncertainties (see in particular Sections 3 and 4). We have discussed some of the aspects that have an important influence on the determination of these parameters and that are relevant for space-based photometry (MOST, CoRoT, Kepler, CHEOPS, EChO, and PLATO), which currently allows the most detailed studies.

\section{References}

[1] J.N. Winn, Exoplanet Transits and Occultations (2011), pp. 55-77

[2] J.T. Wright, B.S. Gaudi, ArXiv e-prints (2012), 1210.2471

[3] K. Mandel, E. Agol, ApJ 580, L171 (2002), arXiv: astro-ph/0210099

[4] Z. Kopal, ed., The Roche problem and its significance for double-star astronomy, Vol. 152 of Astrophysics and Space Science Library (1989)

[5] A. Giménez, A\&A 450, 1231 (2006)

[6] B. Nelson, W.D. Davis, ApJ 174, 617 (1972)

[7] D.M. Popper, P.B. Etzel, AJ 86, 102 (1981)

[8] J. Southworth, P.F.L. Maxted, B. Smalley, MNRAS 351, 1277 (2004), arXiv: astro-ph/0403572

[9] R.E. Wilson, E.J. Devinney, ApJ 166, 605 (1971)

[10] R.E. Wilson, ApJ 234, 1054 (1979)

[11] A.P. Linnell, ApJ 342, 449 (1989)

[12] G. Djurasevic, Ap\&SS 197, 17 (1992)

[13] J.A. Orosz, P.H. Hauschildt, A\&A 364, 265 (2000), arXiv : astro-ph/0010114

[14] A. Prša, T. Zwitter, ApJ 628, 426 (2005), arXiv : astro-ph/0503361

[15] S. Csizmadia, T. Borkovits, Z. Paragi, P. Ábrahám, L. Szabados, L. Mosoni, L. Sturmann, J. Sturmann, C. Farrington, H.A. McAlister et al., ApJ 705, 436 (2009), 0909.5049

[16] D. Kipping, G. Bakos, ApJ 730, 50 (2011), 1004.3538

[17] S. Csizmadia, C. Moutou, M. Deleuil, J. Cabrera, M. Fridlund, D. Gandolfi, S. Aigrain, R. Alonso, J.M. Almenara, M. Auvergne et al., A\&A 531, A41 (2011), 1106.4393

[18] J. Eastman, B.S. Gaudi, E. Agol, ArXiv e-prints (2012), 1206.5798 
[19] A. Collier Cameron, D.M. Wilson, R.G. West, L. Hebb, X.B. Wang, S. Aigrain, F. Bouchy, D.J. Christian, W.I. Clarkson, B. Enoch et al., MNRAS 380, 1230 (2007), 0707.0417

[20] C. Moutou, F. Pont, F. Bouchy, M. Deleuil, J.M. Almenara, R. Alonso, M. Barbieri, H. Bruntt, H.J. Deeg, M. Fridlund et al., A\&A 506, 321 (2009)

[21] H.J. Deeg, M. Gillon, A. Shporer, D. Rouan, B. Stecklum, S. Aigrain, A. Alapini, J.M. Almenara, R. Alonso, M. Barbieri et al., A\&A 506, 343 (2009), 0907.2653

[22] G.Á. Bakos, G. Torres, A. Pál, J. Hartman, G. Kovács, R.W. Noyes, D.W. Latham, D.D. Sasselov, B. Sipőcz, G.A. Esquerdo et al., ApJ 710, 1724 (2010), 0901.0282

[23] T.M. Brown, ApJ 593, L125 (2003), arXiv: astro-ph/0307256

[24] J.M. Almenara, H.J. Deeg, S. Aigrain, R. Alonso, M. Auvergne, A. Baglin, M. Barbieri, P. Barge, P. Bordé, F. Bouchy et al., A\&A 506, 337 (2009), 0908.1172

[25] G. Torres, D.A. Fischer, A. Sozzetti, L.A. Buchhave, J.N. Winn, M.J. Holman, J.A. Carter, ApJ 757, 161 (2012), 1208.1268

[26] J. Ballot, L. Gizon, R. Samadi, G. Vauclair, O. Benomar, H. Bruntt, B. Mosser, T. Stahn, G.A. Verner, T.L. Campante et al., A\&A 530, A97 (2011), 1105. 3551

[27] N.M. Batalha, W.J. Borucki, S.T. Bryson, L.A. Buchhave, D.A. Caldwell, J. ChristensenDalsgaard, D. Ciardi, E.W. Dunham, F. Fressin, T.N. Gautier et al., ApJ 729, 27 (2011), 1102.0605

[28] M. Oshagh, A. Grigahcène, O. Benomar, M.A. Dupret, M.J.P.F.G. Monteiro, R. Scuflaire, N.C. Santos, Successful Asteroseismology for a Better Characterization of the Exoplanet HAT-P-7b, in Advances in Solid State Physics, edited by J.C. Suárez, R. Garrido, L.A. Balona, J. ChristensenDalsgaard (2013), Vol. 31 of Advances in Solid State Physics, p. 227, 1301.0250

[29] D. Valencia, R.J. O’Connell, D. Sasselov, Icarus 181, 545 (2006), arXiv : astro-ph/0511150

[30] C. Sotin, O. Grasset, A. Mocquet, Icarus 191, 337 (2007)

[31] J.J. Fortney, M.S. Marley, J.W. Barnes, ApJ 659, 1661 (2007), arXiv : astro-ph/0612671

[32] S. Seager, M. Kuchner, C.A. Hier-Majumder, B. Militzer, ApJ 669, 1279 (2007), 0707.2895

[33] D. Valencia, D.D. Sasselov, R.J. O’Connell, ApJ 665, 1413 (2007), 0704 . 3454

[34] O. Grasset, J. Schneider, C. Sotin, ApJ 693, 722 (2009), 0902.1640

[35] F.W. Wagner, F. Sohl, H. Hussmann, M. Grott, H. Rauer, Icarus 214, 366 (2011)

[36] J. Southworth, H. Bruntt, D.L. Buzasi, A\&A 467, 1215 (2007), arXiv: astro-ph/0703634

[37] S. Csizmadia, T. Pasternacki, C. Dreyer, J. Cabrera, A. Erikson, H. Rauer, A\&A 549, A9 (2013), 1212.2372

[38] P. Bordé, F. Bouchy, M. Deleuil, J. Cabrera, L. Jorda, C. Lovis, S. Csizmadia, S. Aigrain, J.M. Almenara, R. Alonso et al., A\&A 520, A66 (2010), 1008. 0325

[39] D. Gandolfi, G. Hébrard, R. Alonso, M. Deleuil, E.W. Guenther, M. Fridlund, M. Endl, P. Eigmüller, S. Csizmadia, M. Havel et al., A\&A 524, A55 (2010), 1009. 2597

[40] M. Gillon, A. Hatzes, S. Csizmadia, M. Fridlund, M. Deleuil, S. Aigrain, R. Alonso, M. Auvergne, A. Baglin, P. Barge et al., A\&A 520, A97 (2010), 1007 . 2497

[41] J. Cabrera, H. Bruntt, M. Ollivier, R.F. Díaz, S. Csizmadia, S. Aigrain, R. Alonso, J. Almenara, M. Auvergne, A. Baglin et al., A\&A 522, A110 (2010), 1007.5481

[42] J. Southworth, MNRAS 417, 2166 (2011), 1107.1235

[43] A. Claret, A\&A 506, 1335 (2009)

[44] S.C.C. Barros, D.L. Pollacco, N.P. Gibson, F.P. Keenan, I. Skillen, I.A. Steele, MNRAS 419, 1248 (2012), 1108.3996

[45] W. Hayek, D. Sing, F. Pont, M. Asplund, A\&A 539, A102 (2012)

[46] D.K. Sing, A\&A 510, A21 (2010), 0912.2274

[47] A. Claret, S. Bloemen, A\&A 529, A75 (2011)

[48] H. von Zeipel, MNRAS 84, 665 (1924)

[49] L.W. Twigg, J.B. Rafert, MNRAS 193, 775 (1980)

[50] J.W. Barnes, ApJ 705, 683 (2009), 0909.1752 


\section{Hot Planets and Cool Stars}

[51] F. Espinosa Lara, M. Rieutord, A\&A 533, A43 (2011), 1109.3038

[52] A. Claret, A\&A 538, A3 (2012)

[53] D. Queloz, G.W. Henry, J.P. Sivan, S.L. Baliunas, J.L. Beuzit, R.A. Donahue, M. Mayor, D. Naef, C. Perrier, S. Udry, A\&A 379, 279 (2001), arXiv : astro-ph/0109491

[54] A. Silva-Valio, A.F. Lanza, A\&A 529, A36 (2011), 1102.2192

[55] R. Sanchis-Ojeda, J.N. Winn, ApJ 743, 61 (2011), 1107.2920

[56] J.M. Désert, D. Charbonneau, B.O. Demory, S. Ballard, J.A. Carter, J.J. Fortney, W.D. Cochran, M. Endl, S.N. Quinn, H.T. Isaacson et al., ApJ 197, 14 (2011), 1107.5750

[57] R.J. Jackson, R.D. Jeffries, MNRAS 423, 2966 (2012), 1204.4066 